\title{
Coherence revivals in two-photon frequency combs
}

\author{
Víctor Torres-Company, ${ }^{1,2, *}$ Jesus Lancis, ${ }^{2}$ Hanna Lajunen, ${ }^{3}$ and Ari T. Friberg ${ }^{3,4, \dagger}$ \\ ${ }^{1}$ School of Electrical and Computer Engineering,Purdue University, West Lafayette, Indiana 47907, USA \\ ${ }^{2}$ Departament de Física,Universitat Jaume I, E-12081 Castellon, Spain \\ ${ }^{3}$ Department of Physics and Mathematics, University of Eastern Finland, FI-80101 Joensuu, Finland \\ ${ }^{4}$ Department of Applied Physics,Aalto University, FI-00076 Aalto, Finland
}

(Received 20 April 2011; published 16 September 2011)

\begin{abstract}
We describe and theoretically analyze the self-imaging Talbot effect of entangled photon pairs in the time domain. Rich phenomena are observed in coherence propagation along dispersive media of mode-locked twophoton states with frequency entanglement exhibiting a comblike correlation function. Our results can be used to remotely transfer frequency standards through optical fiber networks with two-photon light, avoiding the requirement of dispersion compensation.
\end{abstract}

DOI: $10.1103 /$ PhysRevA.84.033830

PACS number(s): 42.25.Kb, 42.50.Ar, 42.50.Dv

\section{INTRODUCTION}

Two-photon states can be created through the process of spontaneous parametric down-conversion (SPDC), whereby a photon from a continuous-wave (CW) laser is eventually disintegrated to generate two photons at lower frequencies (referred to as signal and idler) [1]. When the settings of the SPDC process are properly engineered, the down-converted photons can show time-frequency entanglement, revealing that the temporal width of the joint intensity correlation and the individual frequencies of the photons can be measured with accuracies below the minimum uncertainty bound [2]. However, the Fourier rules link the ultrashort nature of the temporal correlations with an ultrawide two-photon spectrum [1]. This broadband spectrum bears intriguing similarities with the properties of ultrashort optical pulses, and many of the techniques that are well established in the field of ultrafast optics [3] can as well be adapted to the two-photon regime (see, e.g., [4-7]).

In this work, we analyze the temporal self-imaging effect, which we show to exist for the two-photon probability amplitude of particular entangled states, that is, so-called twophoton frequency combs $[8,9]$. This effect leads to coherence revivals at periodically spaced effective dispersion values due to an interference process that is analogous to the classical self-imaging phenomenon, the Talbot effect [10]. The basic concepts related to the temporally entangled two-photon states studied in this paper are reviewed in Sec. II and the details of the different self-imaging phenomena are described in Sec. III. Finally, in Sec. IV we discuss the potential applications of the effect and its analogy to classical intensity correlations and present the conclusions.

\footnotetext{
*torres31@ purdue.edu

${ }^{\dagger}$ Also at the Department of Microelectronics and Applied Physics, Royal Institute of Technology (KTH), Electrum 229, SE-164 40 Kista, Sweden.
}

\section{TWO-PHOTON WAVE PACKETS PROPAGATING IN DISPERSIVE MEDIA}

\section{A. Two-photon continuous spectra}

A clear example of the similarity between ultrashort pulses and time-energy entangled photons is shown in Fig. 1. We consider degenerate collinear type II CW-pumped SPDC. The down-converted beams are separated with a polarization beam splitter (PBS) and launched into two different dispersive media. Afterward, the arrival times of all photons are measured with ideal ultrafast detectors and correlated to produce a quantity proportional to the second-order coherence function [11]

$$
G^{(2)}\left(t_{1}, t_{2}\right)=\left\langle\Psi\left|E_{s}^{(-)}\left(t_{1}\right) E_{i}^{(-)}\left(t_{2}\right) E_{i}^{(+)}\left(t_{2}\right) E_{s}^{(+)}\left(t_{1}\right)\right| \Psi\right\rangle,
$$

which depends on $\tau=t_{2}-t_{1}$. In this expression, $E_{s, i}^{( \pm)}(t)$ are the propagated negative- and positive-frequency operators for the signal and idler fields, and $|\Psi\rangle$ is the state generated in the SPDC process. In the two-photon case, we can write $G^{(2)}(\tau)=|\psi(\tau)|^{2}$, where the function $\psi(\tau)$ may be considered as a two-photon probability amplitude that satisfies the Fresnel integral [4],

$$
\psi(\tau)=\int \mathrm{d} \omega \Phi(\omega) \exp \left(i \Phi_{2 \mathrm{eff}} \omega^{2} / 2\right) \exp (-i \omega \tau)
$$

Here, $\Phi(\omega)$ represents the two-photon spectrum, which depends on the phase-matching and crystal parameters, and

$$
\Phi_{2 \mathrm{eff}}=\Phi_{2 s}+\Phi_{2 i}
$$

is the sum of the group delay dispersion (GDD) parameters of the dispersive media in the arms of the signal and idler photons.

The seminal experiments of Valencia et al. [4] provided a clear interpretation for these results: The two-photon probability amplitude propagates analogously to an ultrashort optical pulse in a dispersive medium with equivalent GDD parameter $\Phi_{2 \text { eff }}$ and complex spectrum $\Phi(\omega)$. As shown in Fig. 2, the down-converted photons tend to arrive simultaneously within an uncertainty given by the inverse of the two-photon spectral bandwidth in the absence of dispersion. The two-photon arrival probability distribution broadens significantly when the effective GDD parameter increases. Very often this broadening 


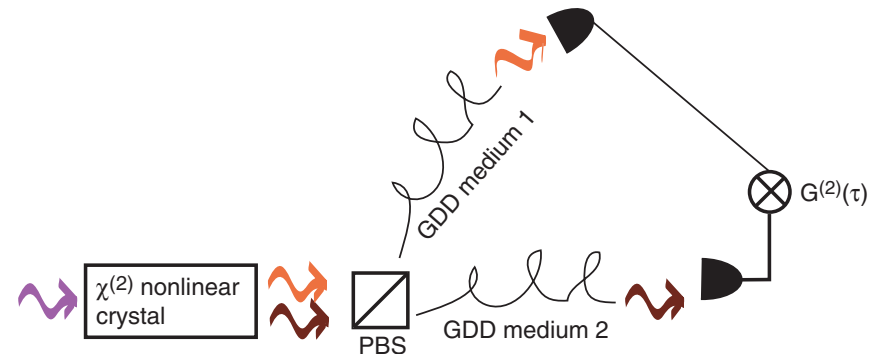

FIG. 1. (Color online) General scheme to study the evolution of a two-photon wave packet when the signal and idler propagate through two different dispersive media. Unless the dispersive properties are properly chosen, the joint probability of two-photon detection gets broadened.

is undesired for applications that are based on two-photon interference [12], becoming more severe in experiments involving the transmission of photons through optical networks, where the GDD parameter is directly proportional to the length of the dispersive fiber. Notably, the shape of the initial $G^{(2)}(\tau)$ can be recovered whenever $\Phi_{2 s}=-\Phi_{2 i}$. This effect is known as dispersion cancellation $[13,14]$ and is interpreted as if the dispersion experienced by one photon is remotely compensated for by the dispersion suffered by its twin.

\section{B. Two-photon frequency combs}

In recent years, interest has grown in a particular case of two-photon states, namely the two-photon frequency combs
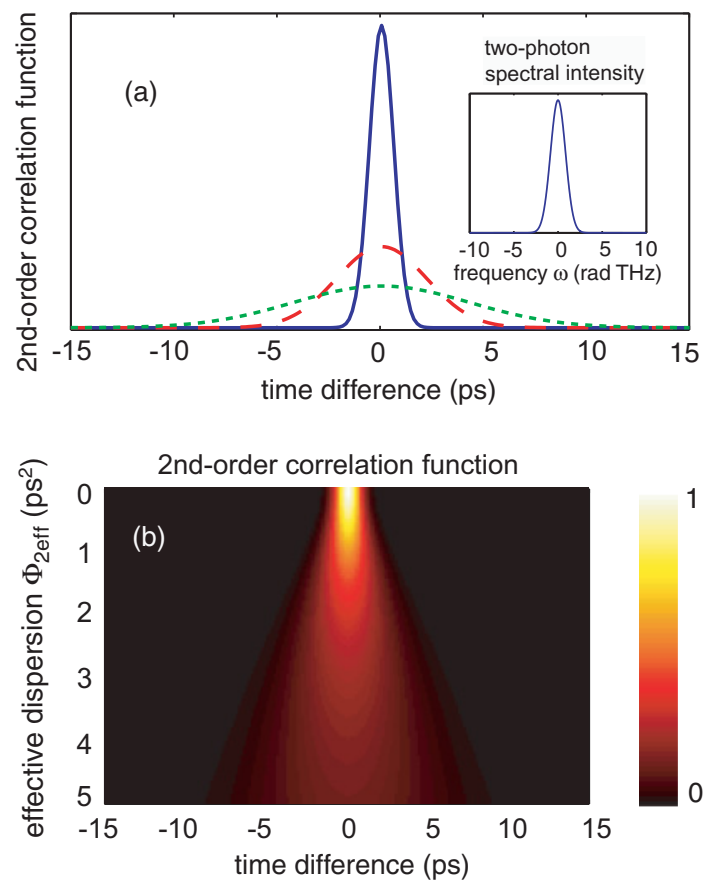

FIG. 2. (Color online) Example of the broadening of the secondorder correlation function $G^{(2)}(\tau)$ of two-photon light with the effective dispersion parameter $\Phi_{\text {2eff }}$. (a) $G^{(2)}(\tau)$ corresponding to a two-photon spectrum (in the inset) with $2-\mathrm{THz}$ full-width halfmaximum (FWHM) bandwidth for $\Phi_{2 \text { eff }}=0 \mathrm{ps}^{2}$ (blue solid line), $2.5 \mathrm{ps}^{2}$ (red long-dashed), and $5.0 \mathrm{ps}^{2}$ (green short-dashed line). (b) Plot of $G^{(2)}(\tau)$ as a function of $\Phi_{2 \text { eff }}$.
$[8,9,15]$. The spectrum of these states consists of evenly spaced narrow peaks modulated by an envelope. Then, the joint probability of two-photon detection shows multiple second-order coherence peaks periodically distributed in time [8]. Following the scheme of Fig. 1, two-photon frequency combs may be implemented by placing an optical cavity in the way of the down-converted collinear photons before they reach the beam splitter. The cavity acts as a periodic spectral filter, leading to the desired comblike structure. Importantly, the resonant cavity might be locked to a frequency reference [16] and thereby transfer its properties to the two-photon mode-locked spectrum [9].

Let us now consider the evolution of this two-photon state through the system of Fig. 1. Figure 3 reveals that, as expected, each of the peaks in the joint probability of two-photon detection broadens with increasing $\Phi_{2 \text { eff }}$. However, we find that the repetitive nature of the joint detection probability can be completely recovered, even when the dispersion is not canceled, for particular GDD parameters which satisfy the relation

$$
\Phi_{2 \mathrm{eff}}=N \frac{T^{2}}{\pi}, \quad N=0,1,2, \ldots
$$

Here $T=2 \pi / \Delta \omega$ is the period of the two-photon coherence and $\Delta \omega$ is the free spectral range of the cavity. We stress that the GDD parameters do not need to be reversed in sign. Revivals of the wave function along the evolution coordinate occur as a natural result of the periodicity in the initial wave form. This is an interference process that can be interpreted in terms of the Talbot effect [10]. It appears as a consequence of wave-packet propagation with dynamics described by a Schrödinger-like equation. Equation (2) predicts the above behavior for the evolution of the two-photon probability amplitude in dispersive media. Actually, Talbot revivals appear in various physical situations, for instance, plasmonics [17], $\mathrm{x}$ rays [18], Bose-Einstein condensates [19], and ultrafast pulses [20]. With two-photon light, spatial Talbot interferences in the two-photon correlation function have also been observed [21] and proposed to coherently phase arrays of quantum emitters in the quest toward intense nonclassical light sources [22].

Figure 4 illustrates the evolution of the joint detection probability, given by the second-order coherence function $G^{(2)}(\tau)$, of two-photon frequency-comb light propagating through a system of two dispersive media with effective GDD

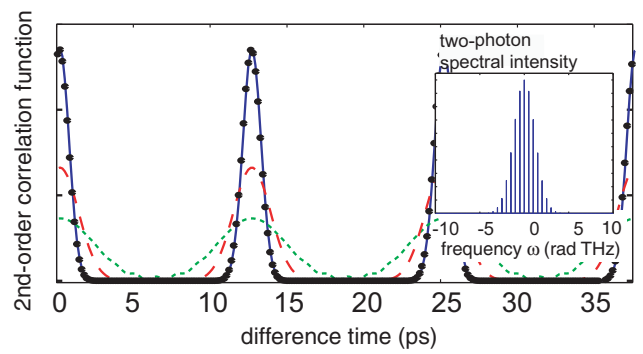

FIG. 3. (Color online) Second-order correlation function $G^{(2)}(\tau)$ corresponding to the two-photon frequency comb filtered by a cavity with the free spectral range of $\Delta \omega=2 \pi \times 80 \mathrm{GHz}$ (in the inset) for $\Phi_{2 \mathrm{eff}}=0 \mathrm{ps}^{2}$ (blue solid line), $1.2 \mathrm{ps}^{2}$ (red long-dashed line), $2.5 \mathrm{ps}^{2}$ (green short-dashed line), and $\Phi_{2 \text { eff }}=T^{2} / \pi \approx 50 \mathrm{ps}^{2}$ (black dots). 

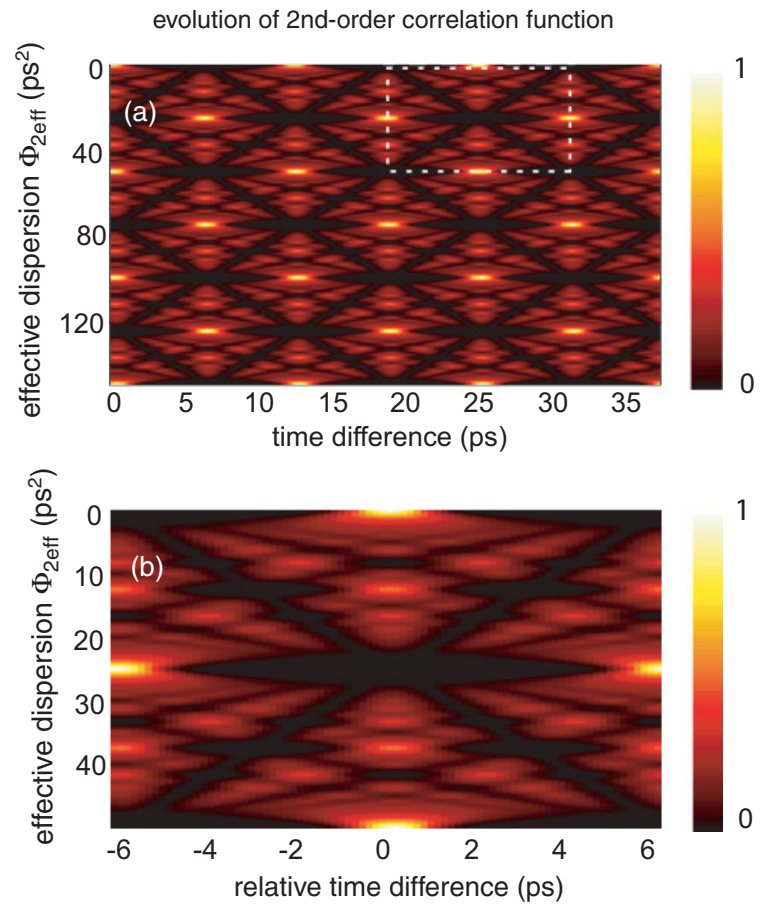

FIG. 4. (Color online) Temporal Talbot carpet. (a) Twodimensional (2D) behavior of second-order coherence with the dispersion parameter. (b) Unit cell.

parameter $\Phi_{2 \text { eff }}$. The dynamics of the propagation makes $G^{(2)}(\tau)$ replicate itself for the GDD parameters provided by Eq. (4) and all the information regarding a single unit cell is contained in the area $[-T / 2 \leqslant \tau \leqslant T / 2],\left[0 \leqslant \Phi_{2 \text { eff }} \leqslant \Phi_{2 T}\right]$, with $\Phi_{2 T}=T^{2} / \pi$, defining what is usually called a Talbot carpet.

\section{TWO-PHOTON TEMPORAL SELF-IMAGING: A QUANTITATIVE DESCRIPTION}

Mathematically, the above characteristics of the two-photon temporal Talbot effect can be described as follows. We consider an ideal two-photon frequency comb with two-photon spectrum

$$
\Phi(\omega)=\phi(\omega) \sum_{n=-\infty}^{+\infty} \delta(\omega-n \Delta \omega) .
$$

This consists of a comb modulated by an envelope. The effect of cavity finesse will be included later. On substituting the above expression into Eq. (2), we get

$$
\begin{aligned}
\psi(\tau)= & \sum_{n=-\infty}^{\infty} \phi(n \Delta \omega) \\
& \times \exp \left[i \Phi_{2 \mathrm{eff}}(n \Delta \omega)^{2} / 2\right] \exp (-i n \Delta \omega \tau) .
\end{aligned}
$$

Now, employing the temporal Talbot condition given by Eq. (4), the propagated two-photon probability amplitude becomes exactly the same as when the GDD parameters are fully compensated for (i.e., $\Phi_{2 \mathrm{eff}}=0$ ), and so

$$
\psi(\tau)=\sum_{n=-\infty}^{\infty} \phi(n \Delta \omega) \exp (-i n \Delta \omega \tau)
$$

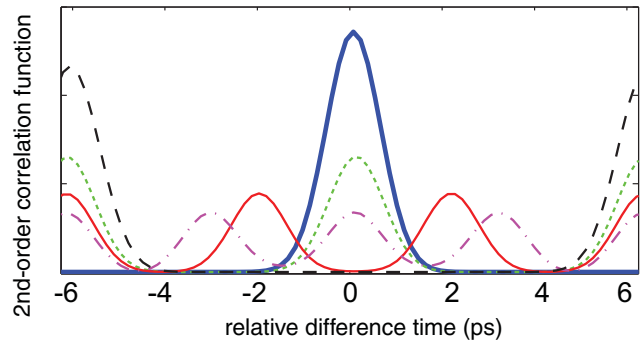

FIG. 5. (Color online) Second-order correlation function $G^{(2)}(\tau)$ for fractional Talbot distances $\Phi_{2 \text { eff }}=0$ (blue solid thick line), $T^{2} / 2 \pi$ (black dashed line), $T^{2} / 4 \pi$ (green short-dashed line), $T^{2} / 6 \pi$ (red solid line), and $T^{2} / 8 \pi$ (magenta dash-dotted line).

Taking a closer look at the details of Fig. 4(a), we observe that the coherence revivals also appear at dispersive parameters satisfying

$$
\Phi_{2 \mathrm{eff}}=\frac{N}{L} \frac{T^{2}}{\pi},
$$

with $N$ and $L$ being coprime integer numbers. However, in this case, the period of the second-order coherence revivals is reduced by a factor $L$. As an example, Fig. 5 illustrates the achieved $G^{(2)}(\tau)$ for some of these particular effective GDD parameters. This effect can be considered as a two-photon equivalent of the fractional Talbot effect [10], which has widely been used in telecommunication systems to increase the repetition rate of mode-locked trains of pulses [23]. We emphasize that the same results are obtained if the envelope is different from the Gaussian, provided the same filling factor is considered. Mathematically, by substituting Eq. (8) into Eq. (2) we find

$$
\psi(\tau)=\sum_{n=-\infty}^{\infty} \phi(n \Delta \omega) \exp \left(i N / L 2 \pi n^{2}\right) \exp (-i n \Delta \omega \tau) .
$$

This equation can further be simplified to [24]

$$
\psi(\tau)=\sum_{m=0}^{L-1} G(m, L, N) \psi_{0}\left(\tau-\frac{m}{L} T\right),
$$

with the coefficients [25]

$$
G(m, L, N)=\frac{1}{L} \sum_{j=0}^{L-1} \exp [-i 2 \pi j(m-j N) / L]
$$

and the function $\psi_{0}(\tau)$ is the two-photon probability amplitude in absence of dispersion [ $\psi(\tau)$ in Eq. (7)].

Equation (10) consists of a sum of delayed and weighted replicas of the input two-photon probability amplitude. Within a fundamental period $T$, a unit cell is shifted $L$ times with different phase factors, thus leading to a decrease in the period of the second-order coherence peaks.

Talbot revivals are affected by the limited finesse of the spectral filter. The spectral lines cannot be properly described by a set of Dirac spikes and the two-photon probability oscillations become damped. In the usual situation, the line width is only a small fraction of the spectral period so that a large number of oscillations are still present. Although wave-packet revivals are no longer sustained in the strict sense due to the limited number of emitters, in this weak-friction 


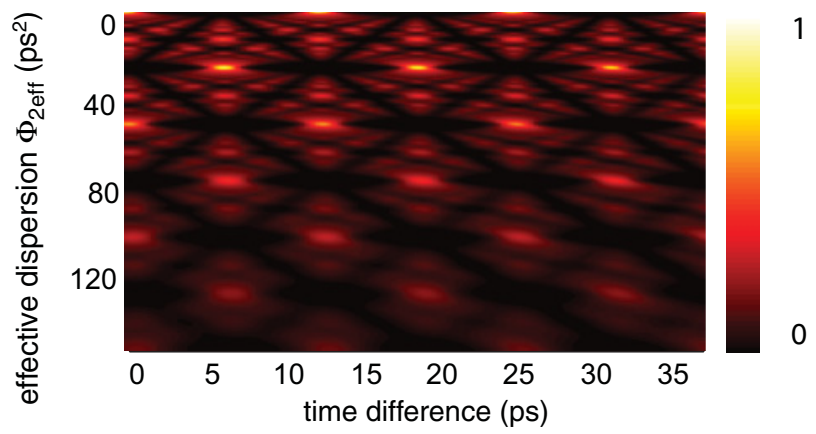

FIG. 6. (Color online) Same Talbot carpet as in Fig. 4(a), but considering a 3-GHz linewdith two-photon frequency comb.

dynamics only a smearing of the unit cell is observed and the phenomenon is nearly conserved. As an example, we recalculate in Fig. 6 the Talbot carpet taking into account the effect of the finite linewidth of the frequency comb. Coherence revivals appear at a limited temporal window and for lower GDD parameters. Similar conclusions were obtained in [26] for the temporal Talbot effect with classical frequency combs of finite linewidth.

\section{CONCLUSIONS}

To conclude, we have shown that the second-order correlation function of two-photon states with a comblike spectrum can recover without the need for dispersion cancellation. This is an interference phenomenon that can be interpreted as the two-photon equivalent of the Talbot effect. We note that this effect is not caused by the unique quantum properties of the light source. Rather, it is due to the fact that the evolution of the two-photon probability amplitude in a dispersive configuration like the one in Fig. 1 satisfies a dynamic equation identical to the diffraction of a monochromatic wave in the paraxial approximation. As such, different light sources, whether quantum or classical, may lead to counterpart coherence revivals. Considering the recently reported fourfold analogy [27] that connects the space-time and quantum-classical domains and recalling that similar Talbot phenomena have been reported with two-photon [21] as well as with classical pseudothermal light $[28,29]$, it is not difficult to envision a similar situation with classical statistically stationary Gaussian light. On the other hand, in the context of classical frequency combs, it has recently been proposed that this effect can be used to transmit frequency standards through optical fibers [30]. Taking into account the facts that two-photon frequency combs may be produced in compact microresonators [15] and these can be locked to an external reference [31], the results presented in this paper open exciting possibilities to remotely transfer frequency standards embedded on ultracompact quantum light sources while circumventing the need for dispersion cancellation.

\section{ACKNOWLEDGMENTS}

This research was supported by the Spanish Government [Projects CSD2007-00013 (SAAUUL) and FIS2010-15746]. Victor Torres-Company is supported by the VII EU Framework Programme through a Marie Curie International Outgoing Fellowship (project PIOF-2009-234996). Ari T. Friberg acknowledges funding from the Academy of Finland.
[1] Y. H. Shih, Rep. Prog. Phys. 66, 1009 (2003).

[2] I. A. Khan and J. C. Howell, Phys. Rev. A 73, 031801 (2006).

[3] A. M. Weiner, Ultrafast Optics (Wiley InterScience, Hoboken, NJ, 2009).

[4] A. Valencia, M. V. Chekhova, A. Trifonov, and Y. Shih, Phys. Rev. Lett. 88, 183601 (2002).

[5] A. Pe'er, B. Dayan, A. A. Friesem, and Y. Silberberg, Phys. Rev. Lett. 94, 073601 (2005).

[6] S. E. Harris, Phys. Rev. Lett. 98, 063602 (2007).

[7] C. Belthangady, C. S. Chuu, I. A. Yu, G. Y. Yin, J. M. Kahn, and S. E. Harris, Phys. Rev. Lett. 104, 223601 (2010).

[8] Y. J. Lu, R. L. Campbell, and Z. Y. Ou, Phys. Rev. Lett. 91, 163602 (2003).

[9] A. Zavatta, V. Parigi, and M. Bellini, Phys. Rev. A 78, 033809 (2008).

[10] K. Patorski, Progr. Opt. 27, 3 (1989).

[11] R. J. Glauber, Phys. Rev. 130, 2529 (1963).

[12] S. Y. Baek, Y. W. Cho, and Y. H. Kim, Opt. Express 17, 19241 (2009).
[13] J. D. Franson, Phys. Rev. A 45, 3126 (1992).

[14] K. A. O’Donnell, Phys. Rev. Lett. 106, 063601 (2011).

[15] J. Chen, Z. H. Levine, J. Fan, and A. L. Migdall, Opt. Express 19, 1470 (2011).

[16] S. T. Cundiff and J. Ye, Rev. Mod. Phys. 75, 325 (2003).

[17] M. R. Dennis, N. I. Zhludev, and F. J. Garcia de Abajo, Opt. Express 15, 9692 (2007).

[18] T. Weitkamp, B. Nohammer, A. Diaz, C. David, and E. Ziegler, Appl. Phys. Lett. 86, 054101 (2005).

[19] L. Deng, E. W. Hagley, J. Denschlag, J. E. Simsarian, M. Edwards, C. W. Clark, K. Helmerson, S. L. Rolston, and W. D. Phillips, Phys. Rev. Lett. 83, 5407 (1999).

[20] P. A. Andrekson, Opt. Lett. 18, 1621 (1993).

[21] K. H. Luo, J. M. Wen, X. H. Chen, Q. Liu, M. Xiao, and L. A. Wu, Phys. Rev. A 80, 043820 (2009).

[22] C. H. R. Ooi and B. L. Lan, Phys. Rev. A 81, 063832 (2010).

[23] J. Azaña and M. A. Muriel, Opt. Lett. 24, 1672 (1999).

[24] V. Arrizón and J. Ojeda Castañeda, Appl. Opt. 33, 5925 (1994).

[25] N. K. Berger, B. Levit, A. Beker, and B. Fischer, IEEE Photon. Technol. Lett. 16, 1855 (2004). 
[26] A. M. Vengsarkar and I. M. Besieris, IEEE Photon. Technol. Lett. 3, 33 (1991).

[27] V. Torres-Company, H. Lajunen, J. Lancis, and A. T. Friberg, Phys. Rev. A 77, 043811 (2008).

[28] K. H. Luo, X. H. Chen, Q. Liu, and L. A. Wu, Phys. Rev. A 82, 033803 (2010).
[29] X. B. Song, J. Xiong, X. Zhang, and K. Wang, Phys. Rev. A 82, 033823 (2010).

[30] H. P. Chuang and C.-B. Huang, Opt. Express 18, 24003 (2010).

[31] P. Del'Haye, O. Arcizet, A. Schliesser, R. Holzwarth, and T. J. Kippenberg, Phys. Rev. Lett. 101, 053903 (2008). 